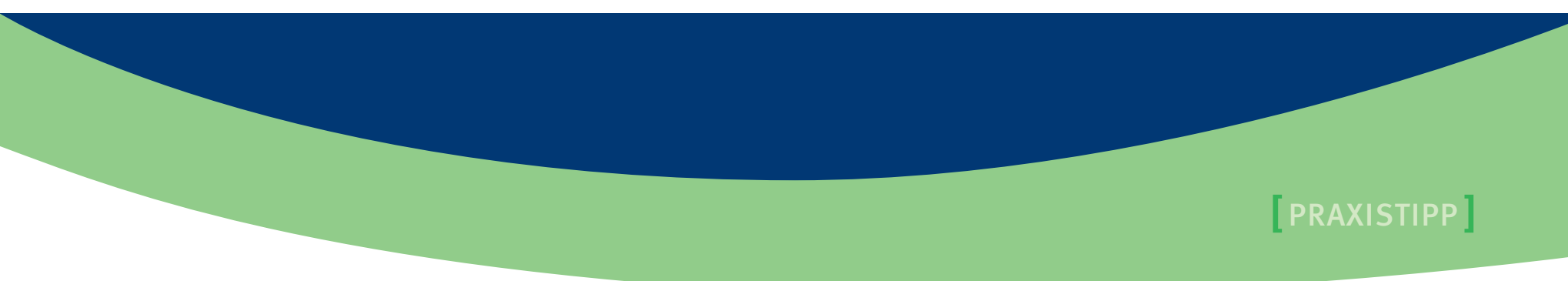

\title{
Rituale in und für Gruppen
}

Gruppenstunden mit mehr als zehn Teilnehmerlnnen können schnell aus dem Ruder geraten. Umso größer eine Gruppe ist, desto dynamischer sind Gruppenprozesse. Kleinigkeiten können den Ablauf so stören, dass der Stundenfluss komplett unterbrochen wird und die Stundenqualität extrem nachlässt. Ablenkungen durch kleine Verletzungen oder Konflikte verunsichern unerfahrene Leitungen. Dies führt dann zu Verzögerungen im Ablauf und hält den Rest der Gruppe auf. Das individuelle Eingehen auf die Teilnehmerlnnen wird somit immer schwieriger. Selbst das sonst so einfache Erklären und Organisieren von Spielen dauert mit einer großen Gruppe länger und kann schnell chaotisch werden. So wird wertvolle Zeit verschwendet, die sinnvoller genutzt werden könnte.

Ein Hilfsmodul für die Gruppenleitung und auch die TeilnehmerInnen können neben einer guten Stundenbild-Vorbereitung auch Rituale sein. Am Beispiel einer Kampfstunde aus dem PAZURU Kampfkunst- und Gesundheitszentrum möchte ich darstellen, wie Rituale den Teilnehmerlnnen und der Leitung helfen können, eine harmonische, hochqualitative Stunde zu erleben. Das Zentrum wurde 2014 in Solingen eröffnet. Der Angebotsschwerpunkt liegt auf einem innovativen Bewegungskonzept für Kinder zwischen drei und 14 Jahren. Das Konzept gliedert sich in die fünf Puzzleteile (Pazuru $=$ jap. für Puzzle) Motorik, Psy- che, Soziales, Ernährung und Natur. Die Kampfkunst wird dabei als Medium zur Charakterschule und Wertevermittlung genutzt (mehr Informationen unter: www.pazuru.de).

Die positive Wirkung von Ritualen kann auf alle Gruppengeschehen übertragen werden: im Kindergarten, in der Schule oder auch zu Hause.

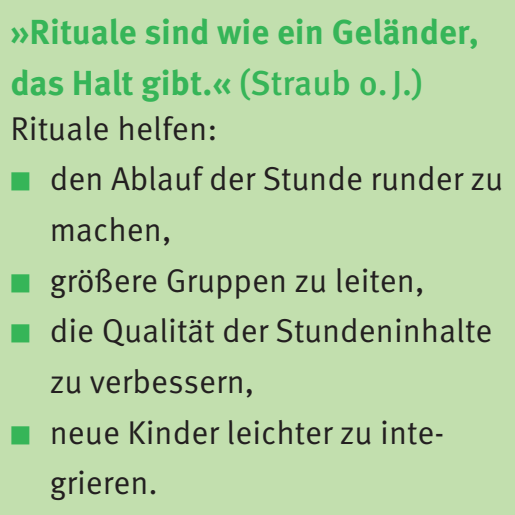

\section{Unterschied Regeln und Rituale}

Regeln sind bestimmte Verkehrsformen, die in Gruppenstunden gelten,
z.B. nicht schlagen oder treten, keine Schimpfwörter benutzen usw.

Rituale gehen über Regeln hinaus. Sie sind ganzheitlicher und werden nicht nur über Sprache vermittelt. Die Vermittlung von Ritualen läuft über Motorik, Emotionen und möglichst viele Sinne (Straub o.J.). Außerdem entfalten Rituale eine bestimmte Symbolkraft, wie zum Beispiel das Verbeugen vor dem Partner in der Kampfkunst (Abb.1). Es bedeutet Respekt und Wertschätzung für den Trainingspartner.

\section{Praxisbeispiel:}

Wenn die Kinder ihre Gürtelprüfung bestehen, werden sie mit ihren Eltern und Verwandten zum Graduierungsevent eingeladen. Dort bekommen sie feierlich von ihren Eltern ihren neuen Gürtel verliehen. Diese Verleihung erfolgt über ein bestimmtes Ritual, bei dem die Eltern und Kinder niederknien und sich verbeugen (Abb.2). Dieser Moment wird von den Eltern als sehr emotional und ergreifend beschrieben.

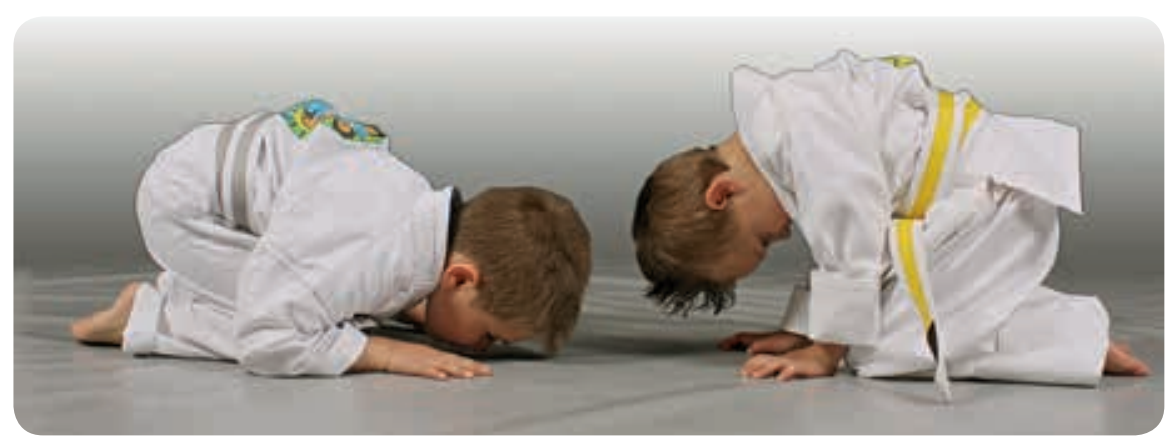

Abb. 1: Verbeugen vor dem Kampf (Foto: Anna Standish-Leigh) 


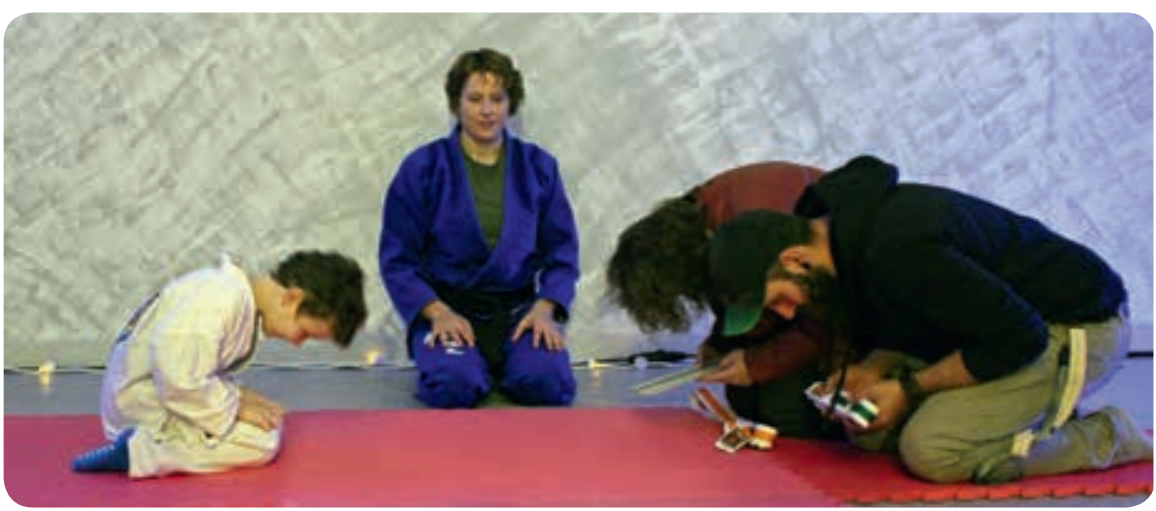

Abb. 2: Graduierungsevent im PAZURU-Kampfkunstzentrum (Foto: Anna Standish-Leigh)

\section{Wie wirken Rituale?}

Rituale beinhalten immer eine Botschaft (Jäggi 2009, 146). Viele Handlungen und Emotionen können nur schwer in Worte gefasst werden, sodass Rituale einen Platzhalter für diese Gefühle darstellen. Rituale wirken nur, wenn sie regelmäßig wiederholt werden (Straub o. J.). Anfangs stellt es eine feste Gewohnheit dar, die dann fließend zu einem Ritual wird. Sobald dieses bekannt ist, muss es nicht jedes Mal neu eingeführt werden. Dadurch können sich die Teilnehmerlnnen auf das Wesentliche konzentrieren.

\section{Rituale und Safe Place}

Der Safe Place ist der innere Halt eines Menschen (Kuntz 2009). Mancherorts wird der innere Safe Place durch einen äußeren Safe Place gefördert, z.B. einen Ort, wo die Teilnehmerlnnen sich jederzeit zurückziehen können, wenn sie ein Bedürfnis nach Ruhe und Sicherheit haben. Solch ein Safe Place, z. B. in Form einer Höhle / abgetrennten Ecke, eignet sich aus meiner Erfahrung nur für Einzel- oder Kleingruppenstunden/-therapie. In Bewegungsstunden mit größeren Gruppen ist solch eine Einrichtung eines Rückzugsortes nicht sinnvoll. Anstatt dessen können aber Rituale Sicherheit geben und dadurch helfen, den inneren Safe Place aufzubauen.

\section{Was spricht für/gegen Rituale?}

\section{PRO - Rituale:}

- bilden einen geschützten Rahmen,

a geben Sicherheit und Halt,

n fördern das Zugehörigkeits-/Gruppengefühl,

- helfen beim Abschalten vom Alltag und

- sorgen für mehr Konzentration auf das Wesentliche.

Das primäre Ziel einer Bewegungsstunde ist, dass die teilnehmenden Kinverbinden. Sie soll einen Ausstieg aus ihrem Alltag darstellen, z. B. wie eine kleine eigene abgeschlossene Welt sein. Kindern, die in schwierigen Familienverhältnissen aufwachsen, kann die regelmäßige Bewegungsstunde Halt im Leben geben - die Lehrkraft als Vorbild und Bezugsperson sowie andere Kinder, die Bestätigung und Anerkennung schenken. Rituale können diesen Ort der Selbstentfaltung abstecken und das »Abschalten« aus dem Alltag fördern und beschleunigen.

In einer Welt, in der alles möglich ist und kaum Grenzen zu existieren scheinen, brauchen Kinder Halt und Orientierung. Denn zu viele Möglichkeiten und Auswahl überfordern einen jungen Menschen. der diese Zeit mit positiven Gefühlen

\section{CONTRA - Rituale:}

- schränken die Kreativität ein,

- schränken die individuelle Entfaltung ein,

- können zu zwanghaftem Verhalten werden und

- können zu automatischen sinnentleerten Mechanismen werden (Zirfas 2004, 10).

In Sekten oder politisch extremistischen Gruppierungen werden Rituale als Machtdemonstration missbraucht. Sobald Rituale zwanghaft, angstmachend und bloßstellend werden, haben sie eine negative Auswirkung auf die Entwicklung (Straub o.J.). Die individuelle Entfaltung, freies Denken und Kreativität werden eingeschränkt und persönliche Interessen unterdrückt.

Auch wenn Rituale nur noch stumpf und automatisch durchgeführt werden, ohne den Sinn zu hinterfragen, wird die Symbolkraft und positive Wirkung aufgehoben. Rituale können auch so eng gesteckt sein, dass keine Entwicklung mehr möglich ist.

\section{Zwischenfazit}

Rituale sollten stets nur einen Rahmen bilden, innerhalb dessen eine individuelle Entwicklung möglich ist. Es sollte regelmäßig in der Gruppe über die Bedeutung der Rituale gesprochen werden, sodass allen TeilnehmernInnen die Sinnhaftigkeit klar ist (Beispiel: Bei jeder Verbeugung sagt der Lehrer $» R e$ spekt, ich pass auf dich auf«, um das Ritual mit dessen Bedeutung verbal zu unterstreichen). Wenn neue Kinder in die Gruppe kommen, ist es wichtig, ein ausführliches Einführungsgespräch (Intro-Stunde) durchzuführen. Nur dann können alle positiven Effekte von Ritualen greifen.

Rituale sind vor allem in großen Gruppen hilfreich. Der Leitung muss allerdings klar sein, dass in großen Grup 
pen immer ein Kompromiss eingegangen werden muss: ein Kompromiss zwischen individuellem Ausleben der eigenen Bedürfnisse und dem sozialen Erleben in der Gruppe durch angeleitete Spiele und Übungen.

\section{Impulse aus dem}

\section{Kampfluunst-Unterricht}

\section{Soziale Rituale unter den Kindern:}

1. Neue Kinder begrüßen: Wenn ein neues Kind am Unterricht teilnimmt, setzt es sich mit in den Begrüßungskreis. Jedes Kind begrüßt dann mit einem »High-Five« das neue Kind und stellt sich mit Namen vor.

2. Mentor-Programm: Während des Begrüßungskreises wird ein freiwilliges Kind gesucht, das das "Vorbild « für das neue Kind ist und die gesamte Stunde an seiner Seite bleibt.

3. Abschluss-Team-Kreis: Am Ende der Unterrichtseinheit legen alle die Hände übereinander und auf drei wird laut »PAZURU « geschrien.

4. Voreinander verbeugen: Vor und nach jedem Übungskampf verbeugen sich die Kinder vor ihrem Partner.

Solche oder ähnliche Rituale können in allgemeinen Gruppenkontexten hilfreich sein, wenn:

neue Kinder schwierig in die Gruppe zu integrieren sind,

- sich kleine Grüppchen bilden, die sich vom Rest der Gruppe isolieren,

a oft Streitigkeiten entstehen,

- der Umgang unter den Kindern nicht so ist, wie die Leitung es sich wünschen würde.

\section{Soziale Rituale Kind-LehrerIn:}

1. Begrüßungskreis am Rand der Matte: 5-10 Minuten vor Beginn der Stunde setzt sich die Lehrperson mit den Kindern an den Mattenrand. Dort ist für alle die Zeit für Geschichten aus dem Kindergarten/der Schule, Urlaubserlebnisse usw.

2. Begrüßung durch »High-Five«: Jedes Kind wird von jeder Lehrperson auf die gleiche Art begrüßt. In Form eines »High Fives« wird Körperkontakt aufgebaut und eine Wertschätzung jedes einzelnen Kindes gezeigt (Abb. 3).

3. Verabschiedung in der Reihe: Am Ende der Stunde laufen alle Lehrerlnnen hintereinander an den Kindern vorbei und verabschieden jeden mit einem »High Five» (Abb.4).

Solche Rituale können in allgemeinen Gruppenkontexten hilfreich sein, wenn:

- die Leitung das Gefühl hat, dass sie durch die Größe der Gruppe nicht mehr jedem einzelnen Kind gerecht wird,

- die Leitung die Bindung zwischen sich und den Kindern stärken möchte.

\section{Rituale im Ablauf (zeitliche Anker- punkte):}

1. An- und Abgrüßungszeremonie: Wie in der Kampfkunst üblich, stellt sich die Gruppe zum An- und Abgrüßen

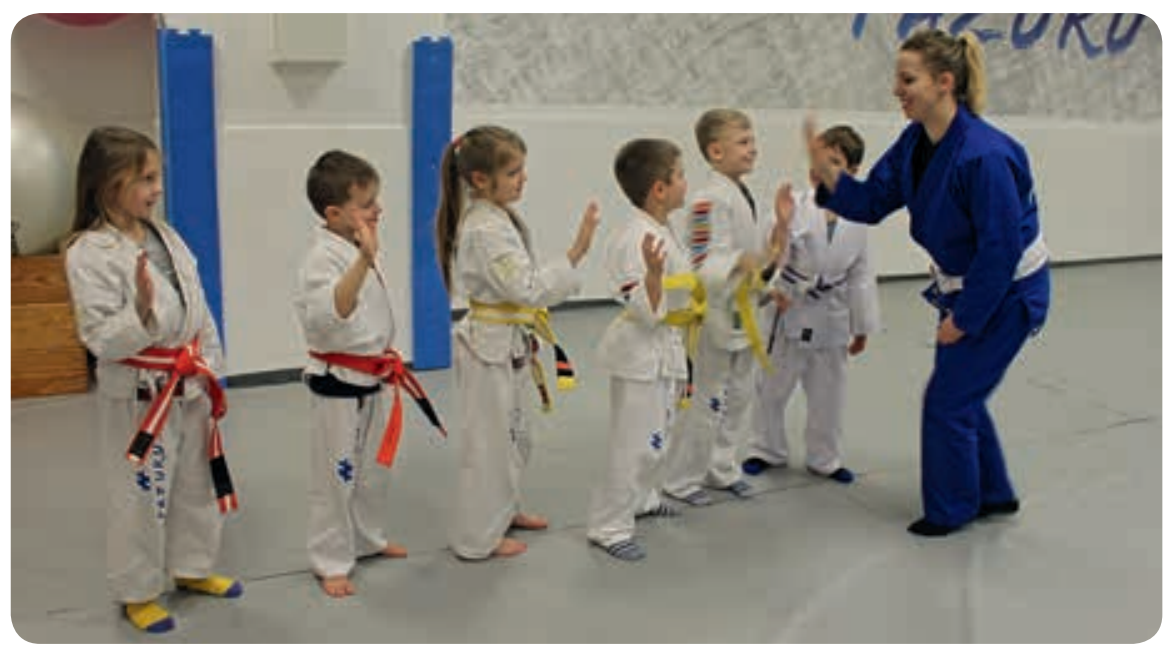

Abb.4: Verabschiedung mit einem »High-Five» (Foto: Ira Paul)

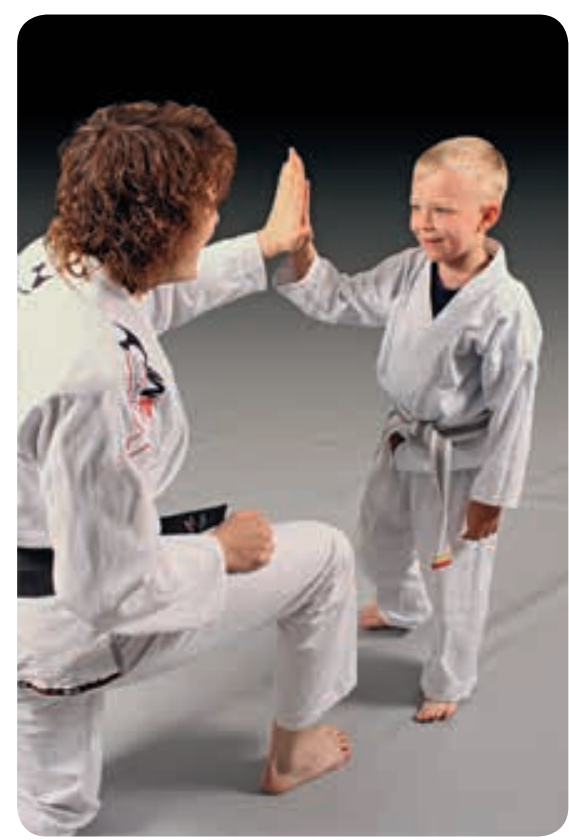

Abb. 3: Begrüßung mit einem »High-Five» (Foto: Anna Standish-Leigh)

in eine Reihe und kniet ab. Nach einer Ruhephase (10-60 Sekunden je nach Alter) verbeugen sich die Kinder und die Lehrerlnnen voreinander.

2. Stunde beginnt immer mit einem Fangspiel: Fangspiele sind einfach zu verstehen, sind dynamisch und verlangen keine Vorkenntnisse. Vorteile: Neue Kinder können sofort mitmachen; wenn Kinder später kommen, wissen sie direkt, welches Spiel läuft; alle Kinder können sich erst einmal auspowern und losrennen.

\section{mot ${ }^{\text {rik }}$}


3. Trinkpause: Es wird eine Trink pause nach immer dem gleichen Programmpunkt (Mattengespräch) durchgeführt. Dadurch weiß jedes Kind, wann die Trinkpause stattfindet.

Solche Rituale können in allgemeinen Gruppenkontexten hilfreich sein, wenn:

- Kinder oft die gleichen Fragen stellen: »Wann ist Trinkpause?«; »Wann machen wir..." usw.,

- sich jede Stunde im Aufbau unterscheidet-ein klarer Stundenaufbau bietet Struktur.

\section{Räumliche Ankerpunkte:}

1. Ort des Begrüßungskreises: Auch wenn die Eltern ihr Kind nur an der Tür abgeben, weiß jedes Kind genau, wo sich alle treffen. Die Kinder kommen nach und nach zum BegrüBungskreis hinzu.

2. Ort für die Gürtel: Der Gürtel ist ein wichtiger Teil der Kampfkunstkleidung. Da der Gürtel schnell zu Boden fällt, bildet er ein Verletzungsrisiko und es kann schnell zu Chaos kommen, wenn jeder seinen Gürtel wieder finden möchte. Deshalb muss jeder, der seinen Gürtel verliert, diesen an die entsprechende Sprosse an der Sprossenwand hängen (Abb. 5). Dies ist auch auf oft genutztes Material übertragbar.

3. Technik-Unterrichts-Punkte: Kleine Punkte in Halbkreisform auf dem Boden, auf denen die Kinder Platz nehmen sollen, bieten Orientierung, wenn Kampfkunsttechniken vermittelt werden. Durch die Punkte können sich auch große Kindergruppen schnell aufstellen. Außerdem wird hier ein bestimmter Ort für Stundeninhalte genutzt, die viel Konzentration erfordern.
4. Mattengesprächspunkte: Diese Punkte sind kreisförmig angeordnet. Dort wird das Mattengespräch zu sozialen Werten (Respekt, Disziplin usw.), Selbstbewusstsein (Mut, Umgang mit Fremden usw.) oder gesunde Ernährung geführt. Dies ist ein Ort für soziale Interaktion.

Solche Rituale können in allgemeinen Gruppenkontexten hilfreich sein, wenn:

- jedes Mal vor Spielerklärungen viel Zeit für die Beruhigung der Gruppe verloren geht,

- Chaos beim Sammeln der Gruppe entsteht (»Macht doch mal einen richtigen Kreis ...»),

- wenn sich die Kinder streiten, wer wo sitzen darf und ggf. manche Kinder keinen Platz mehr haben,

- Material nach der Benutzung chaotisch aufgeräumt wurde oder die Leitung nach der Stunde noch selbst viel Zeit zum Aufräumen aufwenden muss.

\section{Wie führe ich neue Rituale} ein? Was muss ich beachten? Rituale müssen schrittweise eingeübt und regelmäßig praktiziert werden. Das sogenannte Ankern=Einüben dauert einige Zeit. Die Ausübung und Platzierung der Rituale müssen Sie als Kursleitung jedes Mal initiieren. Irgendwann werden die Kinder Sie auch an die Durchführung erinnern, falls Sie es mal vergessen sollten.

Sprechen Sie über das neue Ritual vor oder nach der Stunde. Besetzen Sie das Ritual mit einer bestimmten Sinnhaftigkeit. Reflektieren Sie mit der Gruppe die Emotionen jedes Einzelnen nach diesem neuen Ritual.

Wenn Sie das Gefühl haben, dass die Kinder dieses Ritual nicht gut an-

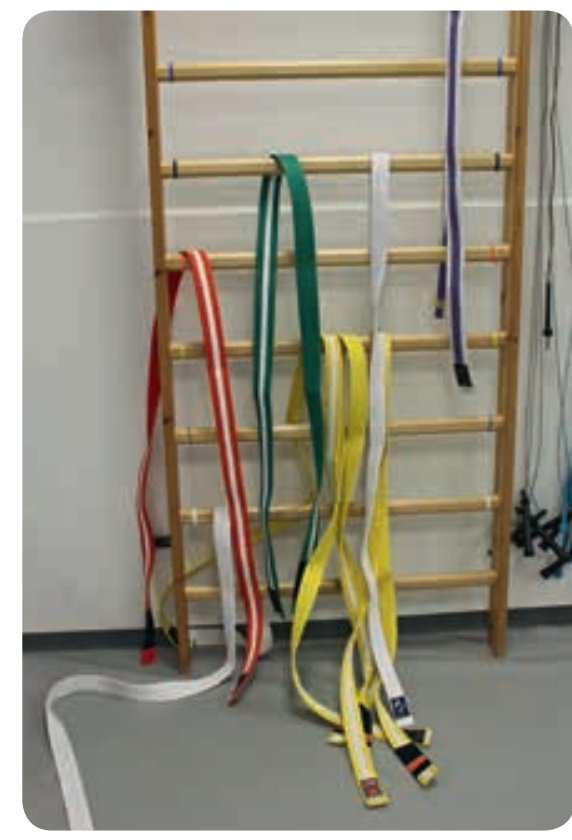

Abb. 5: Gürtel an der Sprossenwand (Foto: Ira Paul)

nehmen, halten Sie nicht verkrampft daran fest. Rituale sind ein Prozess, der sich zwischen den Kindern und Gruppenleitungen entwickelt und sich auch verändern kann.

\section{Literatur}

Jäggi, C. (2009): Sozio-kultureller Code, Rituale und Management. Springer VS, Wiesbaden, https://doi. org/10.1007/978-3-531-91312-4

Kuntz, S. (2009): Der "Safe Place« in der Psychomotorik: Innere und äußere (Sprach-) Räume begegnen sich. motorik 32 (3), 165-175

Straub, C. (o.J.): Die pädagogische Bedeutung von Ritualen. In: http:// www.pb.seminar-albstadt.de/bereiche/paedagogik/paed/rituale.pdf, 20.02.2018

Zirfas, J. (2004): Vom Zauber der Rituale: Der Alltag und seine Regeln. Reclam, Leipzig

Ira Paul

DOI 10.2378/ motorik2018.art24d 